\title{
STRONG LIMITS OF NORMAL OPERATORS
}

\author{
by JOHN B. CONWAY and DONALD W. HADWIN*
}

Dedicated to Paul Halmos on his 65th Birthday

(Received 3 September, 1981)

In [1, Theorem 3.3], E. Bishop proved that an operator $S$ on a Hilbert space $\mathscr{H}$ is subnormal if and only if there is a net of normal operators $\left\{N_{\alpha}\right\}$ that converges to $S$ strongly (that is, $\left\|\left(N_{\alpha}-S\right) f\right\| \rightarrow 0$ for every $f$ in $\mathscr{H}$ ). The proof that such a net exists if $S$ is subnormal is not so difficult; in fact, a sequence of normal operators converging strongly to $S$ can be found. Bishop's proof of the converse, however, is rather complicated and involves, among other things, some complicated arguments using operator-valued measures. The purpose of this note is to provide an easier proof of this part of the theorem. Our interest in finding such a proof was aroused by Paul Halmos.

In [2] and [4] it is shown that an operator $S$ on $\mathscr{H}$ is subnormal if and only if

$$
\sum_{i, j,=0}^{n}\left\langle S^{i} f_{j}, S^{i} f_{i}\right\rangle \geq 0
$$

for every subset $\left\{f_{0}, f_{1}, \ldots, f_{n}\right\}$ of $\mathscr{H}$. It must be shown that if $\left\{N_{\alpha}\right\}$ is a net of normal operators that converges strongly to $S$, then $S$ satisfies $(*)$. If it were the case that $N_{\alpha}^{j} \rightarrow S^{j}$ strongly for all $j \geq 0$, then it would follow that $S$ is subnormal since each $N_{\alpha}$ satisfies $(*)$. However the strong convergence of $\left\{N_{\alpha}\right\}$ to $S$ does not imply the strong convergence of powers of $\left\{N_{\alpha}\right\}$. Consider the following example due to Paul Halmos.

Fix an orthonormal basis $\left\{e_{n}: n \geq 1\right\}$ for $\mathscr{H}$. Since 0 belongs to the weak closure of $\left\{\sqrt{n} e_{n}: n \geq 1\right\}$ ([5, Solution 21]), there is a net of integers $\left\{n_{\alpha}\right\}$ such that $\left\{\sqrt{n_{\alpha}} e_{n_{\alpha}}\right\}$ converges to 0 weakly. If $N_{\alpha}: \mathscr{H} \rightarrow \mathscr{H}$ is defined by $N_{\alpha} f=\sqrt{n_{\alpha}}\left\langle f, e_{n_{\alpha}}\right\rangle e_{n_{\alpha}}$, then each $N_{\alpha}$ is hermitian, $N_{\alpha} \rightarrow 0$ strongly, but $\left\{N_{\alpha}^{2}\right\}$ does not converge strongly to 0 .

The following offers partial relief from these disagreeable affairs.

LEMMA 1. If $\left\{A_{\alpha}\right\}$ and $\left\{B_{\alpha}\right\}$ are nets of operators such that $\sup _{\alpha}\left\|A_{\alpha}\right\|<\infty, A_{\alpha} \rightarrow A$ strongly (respectively, weakly), and $B_{\alpha} \rightarrow B$ strongly, then $A_{\alpha} B_{\alpha} \rightarrow A B$ strongly (respectively, weakly).

The proof of this lemma is an easy exercise.

Note that as a consequence of Lemma 1 if $N_{\alpha} \rightarrow S$ strongly and $\left\{N_{\alpha}\right\}$ is uniformly bounded, then $N_{\alpha}^{j} \rightarrow S^{j}$ strongly for every $j \geq 1$. Thus $(*)$ implies that the strong limit of a bounded net of normal operators is subnormal. Our plan is to reduce the general case to the uniformly bounded case. The principal tool is the following lemma.

\footnotetext{
* This research was partially supported by grants from the National Science Foundation.
}

Glasgow Math. J. 24 (1983) 93-96. 
LEMMA 2. If $\left\{N_{\alpha}: \alpha \in A\right\}$ is a net of normal operators with spectral decompositions $N_{\alpha}=\int_{\mathbb{C}} z d E_{\alpha}(z)$ and $N_{\alpha} \rightarrow S$ strongly, then for any open set $G$ containing $\sigma(S)$, $\left\{E_{\alpha}(G)\right\}$ converges strongly to the identity operator.

Proof. For each $\alpha$ let $Q_{\alpha}=E_{\alpha}(\mathbb{C} \backslash G)$; it must be shown that $Q_{\alpha} \rightarrow 0$ strongly. Equivalently, it must be shown that $Q_{\alpha} \rightarrow 0$ weakly (that is, in the weak operator topology). Since $\left\|Q_{\alpha}\right\|=1$, for all $\alpha$, and the unit ball of $\mathscr{B}(\mathscr{H})$ is weakly compact, $\left\{Q_{\alpha}\right\}$ has a weak cluster point $T$. Let $\left\{Q_{\beta}: \beta \in B\right\}$ be a subnet such that $Q_{\beta} \rightarrow T$ weakly.

Define $u_{\beta}: G \rightarrow \mathscr{B}(\mathscr{H})$ by

$$
u_{\beta}(z)=\int_{C \backslash G}(w-z)^{-1} d E_{\beta}(w)
$$

for each $z$ in $G$. Hence $\left\|u_{\beta}(z)\right\| \leq[\operatorname{dist}(z, \mathbb{C} \backslash G)]^{-1}$ for all $z$ in $G$. So $\left\{u_{\beta}\right\}$ is a locally bounded net of operator-valued analytic functions on $G$. By a version of Montel's theorem for operator-valued analytic functions, there is a function $u: G \rightarrow \mathscr{B}(\mathscr{H})$ such that for all $f$ and $g$ in $\mathscr{H}, z \mapsto\langle u(z) f, g\rangle$ is analytic on $G$ and there is a subnet $\left\{u_{\gamma}: \gamma \in \Gamma\right\}$ of $\left\{u_{\beta}\right\}$ such that $u_{\gamma}(z) \rightarrow u(z)$ weakly for all $z$ in $G$.

Fix $z$ in $G$. Since $\left(N_{\gamma}-z\right) \rightarrow(S-z)$ strongly, Lemma 1 shows that $u_{\gamma}(z)\left(N_{\gamma}-z\right) \rightarrow$ $u(z)(S-z)$ weakly. But $u_{\gamma}(z)\left(N_{\gamma}-z\right)=Q_{\gamma} \rightarrow T$ weakly. Hence $u(z)(S-z)=T$ for all $z$ in $G$. This implies that

$$
f(z)= \begin{cases}u(z) & (z \in G), \\ T(S-z)^{-1} & (z \in \mathbb{C} \backslash \sigma(S))\end{cases}
$$

is a well-defined function from $\mathbb{C}$ into $\mathscr{B}(\mathscr{H})$. Therefore $f$ is an entire function. But $f(z)=T(S-z)^{-1} \rightarrow 0$ as $z \rightarrow \infty$. By Liouville's Theorem, $f$ is constantly 0 . Thus $T=0$.

This argument shows that 0 is the only weak cluster point of the net $\left\{Q_{\alpha}\right\}$. Therefore $Q_{\alpha} \rightarrow 0$ weakly. This completes the proof of Lemma 2 .

The use of Liouville's Theorem in the proof of the preceding lemma is a feature of Bishop's original proof.

Bishop's THEOREM. If $S \in \mathscr{B}(\mathscr{H})$, the following statements are equivalent:

(a) $S$ is subnormal,

(b) $S$ is the strong limit of a net of normal operators.

Proof. The proof that (a) implies (b) is standard. Because of its brevity, the proof is included for completeness. It suffices to assume that $\mathscr{H}$ is separable, so let $\left\{e_{n}: n \geq 1\right\}$ be an orthonormal basis for $\mathscr{H}$. If $N$ is the minimal normal extension of $S$ and $N$ acts on $\mathscr{K}$, then $\mathscr{K}$ is also separable. For each $n \geq 1$, let $U_{n}$ be an isometry from $\mathscr{H}$ onto $\mathscr{K}$ that is the identity on the closed linear span of $\left\{e_{1}, \ldots, e_{n}, S e_{1}, \ldots, S e_{n}\right\}$. Then $U_{n}^{-1} N U_{n}=N_{n} \rightarrow S$ strongly.

Now assume that (b) holds and $\left\{N_{\alpha}\right\}$ is a net of normal operators that converges to $S$ strongly. If $N_{\alpha}=\int_{\mathbb{C}} z d E_{\alpha}(z)$ is the spectral decomposition of $N_{\alpha}$ and $G=$ $\{z \in \mathbb{C}:|z|<\|S\|+1\}$, then Lemma 2 implies $E_{\alpha}(G) \rightarrow 1$ strongly. If $M_{\alpha}=E_{\alpha}(G) N_{\alpha}$, then each $M_{\alpha}$ is normal and, by Lemma $1, M_{\alpha} \rightarrow S$ strongly. Moreover, $M_{\alpha}=\int_{G} z d E_{\alpha}(z)$, so 
that $\left\|M_{\alpha}\right\| \leq\|S\|+1$ for all $\alpha$. Therefore, for each $j \geq 1, M_{\alpha}^{j} \rightarrow S^{i}$ strongly by Lemma 1. By $(*), S$ is subnormal. This completes the proof.

If we restrict ourselves to limits of sequences rather than nets, then we can find analogues of Bishop's theorem for very general classes of operators. Recall that two operators are approximately equivalent if each is a norm limit of operators that are unitarily equivalent to the other. Suppose $\mathscr{S} \subseteq \mathscr{B}(\mathscr{H})$ and $\mathscr{S}$ is closed under unitary equivalence. We say that $S$ is closed under direct sums provided the direct sum of any sequence in $\mathscr{S}$ is unitarily equivalent to an operator in $\mathscr{S}$.

Theorem. Suppose $\mathscr{H}$ is separable, $\mathscr{S} \subseteq \mathscr{B}(\mathscr{H})$, and $\mathscr{S}$ is closed under approximate equivalence and direct sums. If $S \in \mathscr{B}(\mathscr{H})$, then:

(i) $S$ is a strong limit of a sequence in $\mathscr{S}$ if and only if $S$ is unitarily equivalent to the restriction of an operator in $\mathscr{S}$ to an invariant subspace,

(iv) $S$ is a weak limit of a sequence in $\mathscr{S}$ if and only if $S$ is unitarily equivalent to the compression of an operator in $\mathscr{S}$ to some subspace,

(iii) $S$ is the *-strong limit of a sequence in $\mathscr{S}$ if and only if $S$ is unitarily equivalent to the restriction of an operator in $\mathscr{S}$ to a reducing subspace.

Proof. The proof of the "if" parts of (i)-(iii) are similar to the proof of "(a) $\rightarrow$ (b)" in Bishop's theorem above. We prove the "only if" part of (i); the others follow in a similar fashion. Suppose $\left\{S_{n}\right\}$ is a sequence in $\mathscr{S}$ and $S_{n} \rightarrow S$ strongly. Choose $T$ in $\mathscr{S}$ so that $T$ is unitarily equivalent to the direct sum of the $S_{n}$ 's, and choose a sequence $\left\{V_{n}\right\}$ of isometries from $H$ into $H$ so that $V_{n}^{*} T V_{n}=S_{n}$ and $\left(V_{n} V_{n}^{*}\right) T=T\left(V_{n} V_{n}^{*}\right)$ for $n=1,2, \ldots$ It follows from [3, Theorem 3.3, Corollary 3.2] that there is an operator $T_{1}$ that is approximately equivalent to $T$ such that $S$ is unitarily equivalent to the restriction of $T_{1}$ to an invariant subspace. Since $\mathscr{S}$ is closed under approximate equivalence, we know that $T_{1} \in \mathscr{Y}$. This completes the proof.

Remarks. (a). The preceding theorem no longer remains true when "sequence" is replaced by "net". For example, if $\mathscr{S}=\left\{T \in B(H): T^{2}=0\right\}$, then $\mathscr{S}$ is closed under approximate equivalence and direct sums. However, it was shown by Paul Halmos [4, Solution 91] that every operator in $\mathscr{B}(\mathscr{H})$ is a strong limit of a net of operators in $\mathscr{S}$.

(b) Normal operators are direct integrals of 1-dimensional operators. The preceding theorem is false when "sequence" is replaced by net and $\mathscr{S}$ is the set of binormal operators (that is, direct integrals of 1-dimensional and 2-dimensional operators), since the class of binormal operators is known to contain the operators $T$ with $T^{2}=0$. If follows that the analogue of Lemma 2 fails for binormal operators.

\section{REFERENCES}

1. E. Bishop, Spectral theory for operators on a Banach space, Trans. Amer. Math. Soc. 86 (1957), 414-445.

2. J. Bram, Subnormal operators, Duke Math. J. 22 (1955), 75-94.

3. D. Hadwin, Completely positive maps and approximate equivalence, preprint. 
4. P. R. Halmos, Normal dilations and extensions of operators, Summa Brasil. 2 (1950), $125-134$.

5. P. R. Halmos, A Hilbert space problem book (Van Nostrand, 1967).

INDIANA UNIVERSITY

BLOOMINGTON, INDIANA 47405

U.S.A.
University OF New HampshiRe

Durham, New Hampshire 03824

U.S.A. 